\title{
Epilogue for the IJSME Special Issue: Metacognition for Science and Mathematics Learning in Technology-Infused Learning Environments
}

\author{
Ying-Shao Hsu ${ }^{1}$ - Paola Iannone ${ }^{2}$. \\ Hsiao-Ching She ${ }^{3}$ - Allyson F. Hadwin ${ }^{4}$. \\ Larry D. Yore ${ }^{4}$
}

Published online: 4 March 2016

(C) Ministry of Science and Technology, Taiwan 2016

\section{Introduction}

There is some evidence that evolving views of learning (e.g. behaviorism, cognitive development, constructivism, cognitive neuroscience, etc.) over the past five decades have not been fully incorporated into pre-existing subordinate constructs and pedagogical practices (e.g. design of curricula, instructional approaches, roles of teachers, purposes of assessment, etc.) of metacognition-related mathematics and science education research. An example of theory-practice disconnect is constructivism - the preferred view of learning in many mathematics and science education reform documents for the last 25-30 years that is still not fully reflected in classroom instructional (i.e. teaching and assessment) practices. These changes in views of learning have blurred the meanings and boundaries of the foci of this Special Issue: metacognition and self-regulation.

Metacognition still remains inconsistently defined or operationalized as being composed of awareness and self-management. Self-regulated learning - a subcomponent of metacognitive self-management in some definitions of metacognition-was expanded to a multifactor model of learning by Winne and Hadwin (1998). Their COPES model of self-regulated learning (SRL) draws upon a basic cognitive architecture-conditions, operations, products, evaluations, and standards - to explain how metacognitive

Ying-Shao Hsu

yshsu@ntnu.edu.tw

1 National Taiwan Normal University, Taipei, Taiwan

2 University of East Anglia, Norwich, UK

3 National Chiao Tung University, Hsinchu, Taiwan

4 University of Victoria, Victoria, BC, Canada 
monitoring and knowledge dynamically guide strategic action within and across phases of task engagement. Their model emphasizes the role of self, disciplinary, task, and context beliefs as conditions for and products of regulatory cycles. In this way, it has the power to explain the role of metacognition in strategically regulating cognition, motivation, affect, and behavior. Such emerging perspectives emphasize the importance of metacognition and SRL in learning, quality thinking, monitoring, and regulation in dynamic, real-time, complex processes and the hot affective (e.g. motivation, self, identity, etc.) aspects of cognition.

However, in practice, the operational definitions, uses, and measurements of metacognition and SRL constructs in mathematics and science are often fuzzy and fail to consider the role of disciplinary epistemological characteristics and ontological requirements of these domains. Dinsmore, Alexander and Loughlin (2008) suggested that researchers have neglected the clarity of these concepts, have employed instruments without appropriate theoretical roots, and have not reflected clearly on the methodological limitations of such instruments. Some constructs were developed to be generalized across disciplines without fully considering the unique epistemic aspects and ontological requirements of specific domains, for example, the nature of mathematics (NOM) and of science (NOS). Learners' epistemic beliefs about the nature of knowledge and the process of knowing may influence SRL (Hofer \& Sinatra, 2010). Some researchers regard epistemic beliefs as a type of metacognitive knowledge (e.g. Hofer, 2004; Kuhn, 1999), but most popular SRL models integrate epistemic beliefs into the SRL as one component of cognitive conditions (Hsu, Yen, Chang, Wang \& Chen, 2014), for example, the COPES model (Winne \& Hadwin, 1998).

A synthesis of the contents of this Special Issue revealed three major themes flowing from the five articles. These include theoretical perspectives of metacognition and SRL, design issues of metacognitive scaffolding, and methodological approaches used to document metacognition and self-regulation and to examine learning outcomes.

\section{Theoretical Perspectives of Metacognition and Self-regulated Learning}

The differences and confusion between metacognition and self-regulated learning are apparent in the literature and have led to unresolved measurement issues. The early definitions of metacognition generally agreed that it was composed of two clusters: awareness (i.e. declarative knowledge, procedural knowledge, conditional knowledge) and self-management (i.e. setting purpose, selecting and monitoring actions, regulating effort and actions) of cognition. However, difficulties in observing metacognition in all learning situations, measuring real-time self-management of learning, and finding that knowing (awareness) does not ensure use of this knowledge in learning (selfmanagement) limited the support for the theoretical foundations and practical applications of metacognition and SRL in science and mathematics education. Furthermore, there is a lack of metacognition-related and SRL research that explicitly recognizes the epistemic and ontological NOM or NOS in learning about mathematics and science. Fortunately, van Velzen (2015) found very high associations between high school students' explicitness and systematicity of declarative metacognitive knowledge about problem solving and their performance on a series of thought-provoking problem tasks. His preliminary study re-energizes the potential relationships among metacognitive 
awareness (i.e. knowledge of what, how, when, and where), real-time self-management (i.e. executive functions), and achievement in mathematics. However, inconsistent definitions and possible overuse of metacognition as labels for a variety of learning, thinking, reflecting, and non-executive functions have negatively affected its applications in mathematics and science education, similar to those facing engagement that is used to label a wide variety of academic performances, behaviors, approaches, and selfperceptions (Azevedo, 2015).

The fuzzy use of engagement has led to measurement difficulties, which parallels those for metacognition and self-regulation (Sinatra, Heddy \& Lombardi, 2015). Furthermore, the metacognition/self-regulation confusion was exacerbated by the expansion of the self-regulation function to the status of a multifactor SRL model (Winne \& Hadwin, 1998). Pintrich (2000) proposed a taxonomy for SRL that includes four domains (i.e. cognition, motivation, behavior, and context) and four phases (i.e. forethought, monitoring, control and reaction, and reflection). Different SRL models emphasize particular domains or phases and could be expanded to include other factors such as cognitive load (Sweller, 1994), NOM and NOS, sociocultural influences, affective attributes, identity, and so on. For instance, the COPES model (Winne \& Hadwin, 1998) defines four basic phases of the self-regulated process: definition of tasks, goals and plans, study tactics, and adaptation. This model also provides a basis to consider task conditions (e.g. resources, instructional cues, time, and social culture) and cognitive conditions (e.g. beliefs, motivation, domain knowledge, and learning strategies). Science and mathematics education researchers could be well advised to use the COPES or a similar model as a platform on which to consider and explain the epistemic and ontological aspects of the NOS and NOM in SRL environments and tasks.

Based on the COPES model, another theoretical model proposed by Muis (2007) indicates the relation between epistemology and SRL. This model emphasizes that personal epistemology is one component of cognitive conditions, helps learners define the task conditions, and influences their goal setting. Muis believed that personal epistemology translates into epistemic standards as inputs of metacognition, then SRL may influence the development of personal epistemology as a cyclical process.

Different models and their underlying foundational assumptions may cause controversies over key issues related to the studies of metacognition/self-regulated learning such as measurement of SRL (Zimmerman \& Schunk, 2001). For instance, Alpaslan, Yalvac, Loving and Willson (2015) utilized Muis's model to explore the relation among students' personal epistemologies, achievement goals, learning strategies, and achievement using self-report instruments. In contrast, Chen, Huang and Chou's (2015) study applied the COPES model (Winne \& Hadwin, 1998) to design metacognitive scaffolding for experimental goal setting and planning of physics laboratory activities using a questionnaire associated with worksheets to examine learners' goal setting, planning, and self-efficacy. However, none of the articles included in this Special Issue consider the ontological requirements of mathematics or science in discipline-specific learning.

Above all, we would suggest that researchers utilize the notion of multidimensional conceptual framework for the studies of self-regulated actions (Kaplan, 2008). The researchers using this interpretation define self-regulation from their particular theoretical and practical meanings as a set of characteristics among three dimensions: selfaspects, objects of regulation, and strategies for engagement in the task suggested. 
However, the SRL phases in e-learning environments could benefit from an extended empirical-based framework that (a) is constructed using Zimmerman's three-phase model, (b) considers the context of computer-based learning environments, and (c) includes four phases of SRL: task definition and planning, monitoring, control, and reaction and reflection (Devolder, van Braak \& Tondeur, 2012). We recommend using this extended framework to examine scaffold designs and task characteristics of the selected articles. For instance, Chen et al. (2015) designed metacognitive scaffolding for experimental goal setting and planning of physics laboratory activities; they then let the learners select their own goal from a list of experimental goals, justify their choice, decide the procedure of data collection, predict the results, experiment, evaluate, and conclude the findings. These learning tasks belong to the self-aspect, which is regarded as self-determined regulation (Ryan \& Deci, 2000). The aspect of object in this study includes learners' inquiry performance, conceptual understanding, attitude toward science, and self-efficacy. Strategies of engagement in the tasks involved the metacognitive scaffolding associated with probes (e.g. use of tools and technology) that encourages and helps learners to engage and perform learning strategies in physics laboratory.

\section{Cognitive Versus Metacognitive Scaffolding}

Metacognitive scaffolding often aims to remediate learners' awareness and selfmanagement of their learning, while cognitive scaffolding aims to facilitate learning and address learning barriers (Pifarré \& Cobos, 2010; White \& Frederiksen, 1998, 2005; Zimmerman, 2002). The metacognitive scaffolding functions as a guide that reminds learners to reflect on their goals and to find available resources and methods to solve problems (Hannafin, Land \& Oliver, 1999). Three types of mechanism were used as metacognitive scaffolding or metacognitive tools in technology-infused learning environments (Zimmerman \& Tsikalas, 2005):

- Immediate feedback, which could facilitate learners to monitor and evaluate their learning,

- Persistent display of task-related information, which could encourage learners to monitor their learning progress, and

- Collaborative workspaces, which could provide students an indication of their learning status from others' perspectives

Metacognitive scaffolding in general leads learners to monitor their learning process, identify difficulties, and take productive moves or adjustments to reach their learning goals (Zhang, Hsu, Wang \& Ho, 2015). In contrast, cognitive scaffolding was used to help learners overcome their learning difficulties due to their lower-level cognition, such as conceptual understanding, procedural knowledge, and strategies (Davis, 2003; Nuckles, Hubner \& Renkl, 2008).

It is not easy to differentiate metacognitive scaffoldings from learning outcomes because there is a recursive process between them whereby metacognitive processes are linked to cognitive strategies (Winne \& Hadwin, 1998). Metacognitive scaffolding may trigger learners' cognitive performance, and cognitive scaffolding may evoke their 
metacognition. Tang, Wang, Chang, Chen, Lo and Tsai (2015) found that most metacognitive scaffolding is in the form of formative assessments to empower learning including reflective assessment (White \& Frederiksen, 1998) and prompts for reflection (Davis, 2003). They also suggested that the metacognitive scaffolding within technology-infused learning environments often acts as adaptive scaffolding that can be used to support peer-questioning strategies in online collaborative learning (Puntambekar \& Stylianou, 2005) and to support learners' navigation behaviors within hypermedia (Azevedo, Cromley \& Seibert, 2004; Azevedo, Cromley, Winters, Moos \& Greene, 2005).

Two studies reported in this Special Issue used metacognitive scaffoldings in science and mathematics instruction. Chen and Chiu (2015) used collaboration scripts as metacognitive scaffolding, while Chen et al. (2015) applied the COPES model to design metacognitive scaffolding for experimental goal setting and planning. Other studies have also found that the mixture of cognitive and metacognitive scaffolding could enhance learners' cognitive skills (Berthold, Nuckles \& Renkl, 2007; Zhang et al., 2015). Furthermore, a meta-analysis of 33 empirical studies in SRL within computer-based learning environments revealed that the characteristics of learners and the features of tasks (including the types of SRL supports) affect the quality of learners' SRL (Winters, Greene \& Costich, 2008). Therefore, learners' characteristics and task features might be other factors influencing the effects of metacognitive scaffolding on self-regulated actions and cognitive performances.

\section{Methodological Issues}

Methodological issues for metacognition (i.e. metacognitive knowledge and metacognitive skills) or self-regulated actions include methods of assessment, analysis techniques, and data interpretation. Metacognitive knowledge has been assessed by self-report questionnaires with either Likert-type or multiple-choice items, while in comparison, metacognitive skills are investigated by using think-aloud protocols, protocol analysis, and tracing techniques to represent a dynamic, event-based view of metacognition (Winne, Jamieson-Noel \& Muis, 2002; Winne \& Perry, 2000). Exacerbating the problem of assessing metacognitive skills is that not all learning situations will originate observable metacognitive skills because it is generally accepted that metacognitive skills do not become explicit unless the learning is somewhat demanding in terms of the cognitive effort required. That is, metacognition is believed to go underground when it is not needed. However, integrating the cognitive load or demands of an assessment technique, such as think-aloud, onto an already challenging task might overwhelm the learner's cognitive capacity and thereby drive the experience into failure.

The issue of data interpretation is related to which operational definition of metacognition or SRL is selected by the researchers. Metacognition and SRL require a range of measures and methods to capture the multifaceted complexities of these constructs. The methods of assessing and analysis techniques for metacognition or SRL have recently evolved. Tang et al. (2015) revealed that the most current studies (Järvelä \& Hadwin, 2013; Kinnebrew, Segedy \& Biswas, 2014; Malmberg, Järvelä \& Kirschner, 2014; Molenaar, Chiu, Sleegers \& van Boxtel, 2011) utilized 
computation techniques to analyze learners' dialogues or behaviors while being coached in cognitive/metacognitive practices or collaborating in technologyinfused learning environments. These recent studies tried to make a connection between behavior patterns and task sequences/design features. This line of research focuses on the self-regulated actions within scaffolding/guided learning using computing techniques to analyze the recorded qualitative data through the Internet or computers (e.g. online dialogues and behaviors).

Various interpretations and measures of metacognition and SRL were utilized in the other articles in this Special Issue. Researchers collected quantitative data such as selfefficacy (Chen et al., 2015), metacognitive self-regulation (Chen \& Chiu, 2015), and metacognitive strategies (Alpaslan et al., 2015). Qualitative data were also collected and analyzed to support findings such as worksheets (Chen et al., 2015) and open-ended questions (van Velzen, 2015). It is clear from these articles that metacognition and SRL are measured through multiple techniques including self-report surveys, interviews, and open-ended questions. However, utilizing computation techniques to analyze learners' dialogues or behaviors for their self-regulated actions is absent.

van Velzen (2015) addressed the need to document metacognitive knowledge and strategy use within an immediate, demanding, and authentic learning situation. He asked grade 11 students to record their knowledge about mathematics problem solving and strategy use shortly after they solved and discussed ill-structured thought-provoking tasks (TPT). Their responses were stimulated and guided by open-ended questions that promoted reasoning and reflective accounts of their knowledge about problem solving, the problem-solving process, and strategies. The study adopted coding rubrics to the written responses that revealed ordinal categories (4 for explicitness and 5 for systematicity) and scoring rubrics for the TPT solutions. The students' response level correlated highly with their problem-solving performance.

However, the measurements mentioned above (e.g. self-report surveys, interviews, written responses) do not appear to fully address learners' metacognitive awareness, personal control of cognition, and motivation at a microscale. Some scholars have suggested that metacognition and SRL research could benefit from adopting a neuroscience perspective (Fernandez-Duque, Baird \& Posner, 2000; Schwartz, Scott \& Holzberger, 2013) and from analyzing multiple data sources from thinkaloud protocols, eye tracking, and log files to more fully document metacognitive monitoring and control (Azevedo, Moos, Johnson \& Chauncey, 2010; Greene, Dellinger, Tüysüzoğlu \& Costa, 2013). Data mining may help model and study sophisticated metacognition and self-regulation (Baker, Corbett, Roll, Koedinger, Aleven, Cocea \& Hershkovitz, 2013).

It should be noted that no researcher in this Special Issue adopts a neuroscience perspective, partly because utilizing a neuroscience perspective to detect the complex nature of SRL is very challenging and needs multidisciplinary collaboration among researchers, especially for learning tasks involving complicated cognitive processes such as inquiry, problem solving, and argumentation. It is also partly because these researchers view metacognition as an enduring trait and utilize self-report surveys and interviews to assess the aptitude nature of metacognition. See the 2014 Special Issue of the International Journal of Science and Mathematics Education (Anderson, Love \& Tsai, 2014) for recent examples of neuroscience perspectives in science and mathematics education research. 
Winne and Perry (2000) dichotomized the general-specific assumptions underlying metacognition as an aptitude or an event. When viewed as an aptitude, metacognition can be assessed as enduring traits across different tasks (Muis, Winne \& JamiesonNoel, 2007). When metacognition is viewed as an event, metacognitive tactics vary dramatically across different tasks (Pieschl, Stahl, Murray \& Bromme, 2012) and should be examined in real time within a specific task (Moos \& Azevedo 2008). Therefore, researchers measure the general or task-specific features of metacognition under either the aptitude or event nature of metacognition assumption (Wang, 2015). Science and mathematics education researchers need to explore and document the common metacognition and SRL features across disciplines and topics, while being receptive and alert to the unique metacognitive and SRL features inherent in science or mathematics because of their discipline-specific epistemic and ontological aspects. In sum, metacognition is complex and multifaceted; therefore, researchers utilize different types of measurements to disclose different features or triangulate across several measurements to better represent informed views of learning and the learners' selfregulated process. It is also noted that different methods or techniques of measurement used in empirical studies reveal the researchers' underlying assumptions about metacognition. The mathematics and science education research communities would be well served by a web-based repository of verified metacognition measures for different views of learning and tasks and topics within mathematics and science learning.

\section{Conclusion and Future Studies}

An emerging research trend for studying self-regulated actions is to explore learners' behavior patterns through analyzing the dynamic and complex interactions between monitoring and controlling processes during SRL using computing technique (Tang et al., 2015). Therefore, future research directions should focus more on (a) what adaptive scaffoldings support self-regulated actions, (b) how characteristics and features of adaptive scaffolding for SRL facilitate various learning performances, (c) what patterns of students' self-regulated actions are evident while students react to adaptive scaffolding through utilizing computing techniques, and (d) metacognitive scaffolding in technology-infused environments.

Mathematics and science learning, especially SRL, involves a complex interplay of learner, task, discipline, and sociocultural factors. Researchers need to consider a broad range of personal traits or conditions that learners bring to the task including prior knowledge and experiences, cultural beliefs and values, epistemology, motivation, interest, self-efficacy, disciplinary identity and self-concept, linguistic resources, and alignment with languages of home, instruction, and discipline (i.e. mathematics or science language and metalanguage or enterprise language). There is a need for an integrated view of learning, teaching, and assessment in mathematics and science education at all levels of schooling when examining metacognition and SRL. Central to this integration is the realization that many constructs (e.g. critical thinking, reflection, metacognition) dealing with mathematics and science learning were developed from different philosophies of education and views of cognition (e.g. progressive education, behaviorism, cognitive development, etc.) that converge under newer views (e.g. constructivism, cognitive science, neuroscience) of learning and teaching (Ford \& Yore, 2012). 


\section{References}

Alpaslan, M.M., Yalvac, B., Loving, C.C. \& Willson, V. (2015). Exploring the relationship between high school students' physics-related personal epistemologies and self-regulated learning in Turkey. International Journal of Science and Mathematics Education. doi:10.1007/s10763-015-9685-7.

Anderson, O. R., Love, B. C. \& Tsai, M.-J. (Eds.). (2014). Neuroscience perspectives for science and mathematics learning in technology-enhanced learning environments [Special Issue]. International Journal of Science and Mathematics Education, 12(3), 467-703.

Azevedo, R. (2015). Defining and measuring engagement and learning in science: conceptual, theoretical, methodological, and analytical issues. Educational Psychologist, 50(1), 84-94. doi:10.1080/00461520. 2015.1004069.

Azevedo, R., Cromley, J. G., Winters, F. I., Moos, D. C. \& Greene, J. A. (2005). Adaptive human scaffolding facilitates adolescents' self-regulated learning with hypermedia. Instructional Science, 33(5-6), 381-412. doi:10.1007/s11251-005-1273-8.

Azevedo, R., Cromley, J. G. \& Seibert, D. (2004). Does adaptive scaffolding facilitate students' ability to regulate their learning with hypermedia? Contemporary Educational Psychology, 29(3), 344-370. doi:10. 1016/j.cedpsych.2003.09.002.

Azevedo, R., Moos, D. C., Johnson, A. M. \& Chauncey, A. D. (2010). Measuring cognitive and metacognitive regulatory process during hypermedia learning: issues and challenges. Educational Psychologist, 45(4), 201-223. doi:10.1080/00461520.2010.515934.

Baker, R.S.J.d., Corbett, A.T., Roll, I., Koedinger, K.R., Aleven, V., Cocea, .. Mathews, M. (2013). Modeling and studying gaming the system with educational data mining. In R. Azevedo \& V. Aleven (Eds.), International handbook of metacognition and learning technologies (Vol. 28, pp. 97-115). New York, NY: Springer. doi:10.1007/978-1-4419-5546-3 6.

Berthold, K., Nuckles, M. \& Renkl, A. (2007). Do learning protocols support learning strategies and outcomes? The role of cognitive and metacognitive prompts. Learning and Instruction, 17, 564-577. doi:10.1016/j.learninstruc.2007.09.007.

Chen, C.-H. \& Chiu, C.-H. (2015). Collaboration scripts for enhancing metacognitive self-regulation and mathematics literacy. International Journal of Science and Mathematics Education. doi:10.1007/s10763015-9681-y.

Chen, S., Huang, C.-C. \& Chou, T.-L. (2015). The effect of metacognitive scaffolds on low achievers' laboratory learning. International Journal of Science and Mathematics Education. doi:10.1007/s10763015-9691-9.

Davis, E. A. (2003). Prompting middle school science students for productive reflection: generic and directed prompts. Journal of the Learning Sciences, 12(1), 91-142. doi:10.1207/S15327809JLS1201_4.

Devolder, A., van Braak, J. \& Tondeur, J. (2012). Supporting self-regulated learning in computer-based learning environments: systematic review of effects of scaffolding in the domain of science education. Journal of Computer Assisted Learning, 28(6), 557-573. doi:10.1111/j.1365-2729.2011.00476.x.

Dinsmore, D. L., Alexander, P. A. \& Loughlin, S. M. (2008). Focusing the conceptual lens on metacognition, self-regulation, and self-regulated learning. Educational Psychology Review, 20(4), 391-409. doi:10. 1007/s10648-008-9083-6.

Fernandez-Duque, D., Baird, J. A. \& Posner, M. I. (2000). Executive attention and metacognitive regulation. Consciousness and Cognition, 9(2), 288-307. doi:10.1006/cog.2000.0447.

Ford, C. L. \& Yore, L. D. (2012). Toward convergence of metacognition, reflection, and critical thinking: Illustrations from natural and social sciences, teacher education, and classroom practice. In A. Zohar \& Y. J. Dori (Eds.), Metacognition in science education: Trends in current research (Vol. 40, pp. 251-271). Dordrecht, The Netherlands: Springer.

Greene, J. A., Dellinger, K. R., Tüysüzoğlu, B. B. \& Costa, L.-J. (2013). A two-tiered approach to analyzing self-regulated learning data to inform the design of hypermedia learning environments. In R. Azevedo \& V. Aleven (Eds.), International handbook of metacognition and learning technologies (Vol. 28, pp. 117127). New York, NY: Springer. doi:10.1007/978-1-4419-5546-3_6.

Hannafin, M. J., Land, S. \& Oliver, K. (1999). Open learning environments: Foundations and models. In C. Reigeluth (Ed.), Instructional design theories and models: A new paradigm of instructional theory (pp. 115-140). Mahwah, NJ: Erlbaum.

Hofer, B. K. (2004). Paradigmatic approaches to personal epistemology. Educational Psychologist, 39(1), 1-3. doi:10.1207/s15326985ep3901_1.

Hofer, B. K. \& Sinatra, G. M. (2010). Epistemology, metacognition, and self-regulation: musings on an emerging field. Metacognition and Learning, 5(1), 113-120. doi:10.1007/s11409-009-9051-7. 
Hsu, Y.-S., Yen, M.-H., Chang, W.-H., Wang, C.-Y. \& Chen, S. (2014). Content analysis of 1998-2012 empirical studies in science reading using a self-regulated learning lens. International Journal of Science and Mathematics Education. doi:10.1007/s10763-014-9574-5.

Järvelä, S. \& Hadwin, A. (2013). New frontiers: regulating learning in CSCL. Educational Psychologist, 48(1), 25-39. doi:10.1080/00461520.2012.74800.

Kaplan, A. (2008). Clarifying metacognition, self-regulation, and self-regulated learning: what's the purpose? Educational Psychology Review, 20(4), 477-484. doi:10.1007/s10648-008-9087-2.

Kinnebrew, J. S., Segedy, J. R. \& Biswas, G. (2014). Analyzing the temporal evolution of students' behaviors in open-ended learning environments. Metacognition and Learning, 9(2), 187-215. doi:10.1007/s11409014-9112-4.

Kuhn, D. (1999). A developmental model of critical thinking. Educational Researcher, 28(2), 16-25. doi:10. 3102/0013189x028002016.

Malmberg, J., Järvelä, S. \& Kirschner, P. (2014). Elementary school students' strategic learning: does task-type matter? Metacognition and Learning, 9(2), 113-136. doi:10.1007/s11409-013-9108-5.

Molenaar, I., Chiu, M. M., Sleegers, P. \& van Boxtel, C. (2011). Scaffolding of small groups' metacognitive activities with an avatar. International Journal of Computer-Supported Collaborative Learning, 6(4), 601-624. doi:10.1007/s11412-011-9130-z.

Moos, D. C. \& Azevedo, R. (2008). Exploring the fluctuation of motivation and use of self-regulatory processes during learning with hypermedia. Instructional Science, 36(3), 203-231. doi:10.1007/s11251007-9028-3.

Muis, K. R. (2007). The role of epistemic beliefs in self-regulated learning. Educational Psychologist, 42(3), 173-190. doi:10.1080/00461520701416306.

Muis, K. R., Winne, P. H. \& Jamieson-Noel, D. (2007). Using multitrait-multimethod analysis to examine conceptual similarities of three self-regulated learning inventories. British Journal of Educational Psychology, 77(1), 177-195. doi:10.1348/000709905X90876.

Nuckles, M., Hubner, S. \& Renkl, A. (2008). Short-term versus long-term effects of cognitive and metacognitive prompts in writing-to-learn. In G. Kanselaar, V. Jonker, P. A. Kirschner, \& F. J. Prins (Eds.), Proceedings of the 8th International Conference of the Learning Sciences (Vol. 2, pp. 124-131). Utrecht, NL: ICLS.

Pieschl, S., Stahl, E., Murray, T. \& Bromme, R. (2012). Is adaptation to task complexity really beneficial for performance? Learning and Instruction, 22(4), 281-289. doi:10.1016/j.learninstruc.2011.08.005.

Pifarré, M. \& Cobos, R. (2010). Promoting metacognitive skills through peer scaffolding in a CSCL environment. International Journal of Computer-Supported Collaborative Learning, 5(2), 237-253. doi:10.1007/s11412-010-9084-6.

Pintrich, P. R. (2000). The role of goal orientation in self-regulated learning. In M. Boekaerts, P. R. Pintrich \& M. Zeidner (Eds.), Handbook of self-regulation (pp. 451-502). San Diego, CA: Academic.

Puntambekar, S. \& Stylianou, A. (2005). Designing navigation support in hypertext systems based on navigation patterns. Instructional Science, 33(5), 451-481. doi:10.1007/s11251-005-1276-5.

Ryan, R. M. \& Deci, E. L. (2000). Self-determination theory and the facilitation of intrinsic motivation, social development, and well-being. American Psychologist, 55(1), 68-78. doi:10.1037/0003-066X.55.1.68.

Schwartz, N. H., Scott, B. M. \& Holzberger, D. (2013). Metacognition: A closed-loop model of biased competition-evidence from neuroscience, cognition, and instructional research. In R. Azevedo \& V. Aleven (Eds.), International handbook of metacognition and learning technologies (Vol. 28, pp. 79-94). New York, NY: Springer. doi:10.1007/978-1-4419-5546-3_6.

Sinatra, G. M., Heddy, B. C. \& Lombardi, D. (2015). The challenges of defining and measuring student engagement in science. Educational Psychologist, 50(1), 1-13. doi:10.1080/00461520.2014.1002924.

Sweller, J. (1994). Cognitive load theory, learning difficulty, and instructional design. Learning and Instruction, 4(4), 295-312. doi:10.1016/0959-4752(94)90003-5.

Tang, K.-Y., Wang, C.-Y., Chang, H.-Y., Chen, S., Lo, H.-C. \& Tsai, C.-C. (2015). The intellectual structure of metacognitive scaffolding in science education: a co-citation network analysis. International Journal of Science and Mathematics Education. doi:10.1007/s10763-015-9696-4.

van Velzen, J. H. (2015). Eleventh-grade high-school students' accounts of mathematical metacognitive knowledge: explicitness and systematicity. International Journal of Science and Mathematics Education. doi:10.1007/s10763-015-9689-3.

Wang, C.-Y. (2015). Exploring general versus task-specific assessments of metacognition in university chemistry students: a multitrait-multimethod analysis. Research in Science Education, 45(4), 555-579. doi:10.1007/s11165-014-9436-8.

White, B. Y. \& Frederiksen, J. R. (1998). Inquiry, modeling, and metacognition: making science accessible to all students. Cognition and Instruction, 16(1), 3-118. doi:10.1207/s1532690xci1601_2. 
White, B. Y. \& Frederiksen, J. R. (2005). A theoretical framework and approach for fostering metacognitive development. Educational Psychologist, 40(4), 211-223. doi:10.1207/s15326985ep4004_3.

Winne, P. H. \& Hadwin, A. F. (1998). Studying as self-regulated learning. Mahwah, NJ: Lawrence Erlbaum.

Winne, P. H., Jamieson-Noel, D. \& Muis, K. R. (2002). Methodological issues and advances in researching tactics, strategies, and self-regulated learning. In P. R. Pintrich \& M. L. Maehr (Eds.), Advances in motivation and achievement: New directions in measures and methods (Vol. 12, pp. 121-155). Greenwich, CT: JAI Press.

Winne, P. H. \& Perry, N. E. (2000). Measuring self-regulated learning. In M. Boekaerts, P. R. Pintrich \& M. Zeidner (Eds.), Handbook of self-regulation (pp. 531-566). San Diego, CA: Academic. doi:10.1016/ B978-012109890-2/50045-7.

Winters, F., Greene, J. \& Costich, C. (2008). Self-regulation of learning within computer-based learning environments: a critical analysis. Educational Psychology Review, 20(4), 429-444. doi:10.1007/s10648008-9080-9.

Zhang, W.-X., Hsu, Y.-S., Wang, C.-Y. \& Ho, Y.-T. (2015). Exploring the impacts of cognitive and metacognitive prompting on students scientific inquiry practices within an e-learning environment. International Journal of Science Education, 37(3), 529-553. doi:10.1080/09500693.2014.996796.

Zimmerman, B. J. (2002). Becoming a self-regulated learner: an overview. Theory Into Practice, 41(2), 64-70. doi:10.1207/s15430421tip4102_2.

Zimmerman, B. J. \& Schunk, D. H. (Eds.). (2001). Self-regulated learning and academic achievement: Theoretical perspectives. Hillsdale, NJ: Erlbaum.

Zimmerman, B. J. \& Tsikalas, K. E. (2005). Can computer-based learning environments (CBLEs) be used as self-regulatory tools to enhance learning? Educational Psychologist, 40(4), 267-271. doi:10.1207/ s15326985ep4004_8. 